\title{
Correction: Constructing High-Fidelity Phenotype Knowledge Graphs for Infectious Diseases With a Fine-Grained Semantic Information Model: Development and Usability Study
}

Lizong Deng ${ }^{1,2^{*}}$, PhD; Luming Chen ${ }^{1,2^{*}}$, MS; Tao Yang ${ }^{1,2^{*}}$, MM; Mi Liu ${ }^{3}$ PhD; Shicheng Li $^{1,2}$, MM; Taijiao Jiang ${ }^{1,2,4}$, $\mathrm{PhD}$

${ }^{1}$ Center of Systems Medicine, Institute of Basic Medical Sciences, Chinese Academy of Medical Sciences \& Peking Union Medical College, Beijing, China

${ }^{2}$ Suzhou Institute of Systems Medicine, Suzhou, China

${ }^{3}$ Jiangsu Institute of Clinical Immunology, Jiangsu Key Laboratory of Clinical Immunology, The First Affiliated Hospital of Soochow University, Suzhou, China

${ }^{4}$ Guangzhou Laboratory, Guangzhou, China

*these authors contributed equally

\section{Corresponding Author:}

Taijiao Jiang, $\mathrm{PhD}$

Center of Systems Medicine

Institute of Basic Medical Sciences

Chinese Academy of Medical Sciences \& Peking Union Medical College

\#5 Dong Dan San Tiao

Dongcheng District

Beijing, 100005

China

Phone: 86051262873781

Email: taijiao@ibms.pumc.edu.cn

\section{Related Article:}

Correction of: https://www.jmir.org/2021/6/e26892

(J Med Internet Res 2021;23(7):e31481) doi: 10.2196/31481

In "Constructing High-Fidelity Phenotype Knowledge Graphs for Infectious Diseases With a Fine-Grained Semantic Information Model: Development and Usability Study" (J Med Internet Res 2021;23(6):e26892) the authors noted three errors.

1. In the originally published manuscript, affiliation 1,2 , and 3 were incorrectly mentioned as follows:

${ }^{1}$ Suzhou Institute of Systems Medicine, Suzhou, China

${ }^{2}$ Jiangsu Institute of Clinical Immunology, Jiangsu Key Laboratory of Clinical Immunology, The First Affiliated Hospital of Soochow University, Suzhou, China

${ }^{3}$ Center of Systems Medicine, Institute of Basic Medical Sciences, Chinese Academy of Medical Sciences \& Peking Union Medical College, Beijing, China

These have been corrected to the following:

${ }^{1}$ Center of Systems Medicine, Institute of Basic Medical Sciences, Chinese Academy of Medical Sciences \& Peking Union Medical College, Beijing, China

\footnotetext{
${ }^{2}$ Suzhou Institute of Systems Medicine, Suzhou, China

${ }^{3}$ Jiangsu Institute of Clinical Immunology, Jiangsu Key Laboratory of Clinical Immunology, The First Affiliated Hospital of Soochow University, Suzhou, China
}

2. The affiliation "Center of Systems Medicine, Institute of Basic Medical Sciences, Chinese Academy of Medical Sciences \& Peking Union Medical College, Beijing, China" was originally listed only for author Taijiao Jiang. This affiliation has now been added to authors Lizong Deng, Luming Chen, Tao Yang, and Shicheng $\mathrm{Li}$ in addition to author Taijiao Jiang.

3. The address of the corresponding author was originally published as follows:

\section{Chongwen Road 100 \\ Suzhou Industrial Park \\ Suzhou, 215000 \\ China}

This has been corrected to the following:

\#5 Dong Dan San Tiao

Dongcheng District 
Beijing, 100005

China

The correction will appear in the online version of the paper on the JMIR Publications website on July 13, 2021, together with the publication of this correction notice. Because this was made after submission to PubMed, PubMed Central, and other full-text repositories, the corrected article has also been resubmitted to those repositories.

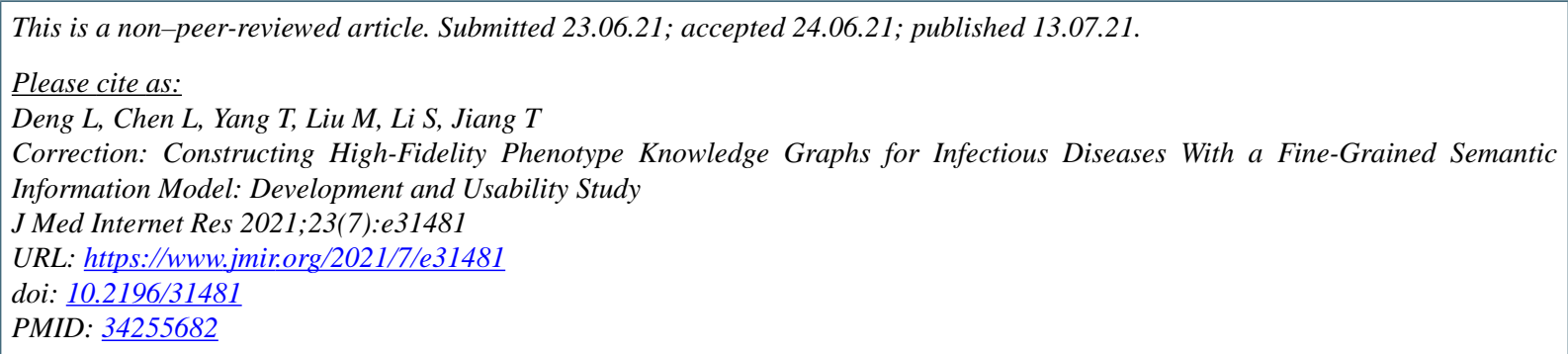

(CLizong Deng, Luming Chen, Tao Yang, Mi Liu, Shicheng Li, Taijiao Jiang. Originally published in the Journal of Medical Internet Research (https://www.jmir.org), 13.07.2021. This is an open-access article distributed under the terms of the Creative Commons Attribution License (https://creativecommons.org/licenses/by/4.0/), which permits unrestricted use, distribution, and reproduction in any medium, provided the original work, first published in the Journal of Medical Internet Research, is properly cited. The complete bibliographic information, a link to the original publication on https://www.jmir.org/, as well as this copyright and license information must be included. 